\title{
SUPLEMENTOS NUTRICIONAIS
}

\section{NUTRITIONAL SUPLLEMENTS}

Tatiana Munhoz da Rocha Lemos Costa'; Victoria Zeghbi Cochenski Borba

\section{RESUMO}

A promessa de resultados rápidos e milagrosos no ganho de massa muscular, definição corporal, redução de gordura e peso corporal vem contribuindo para o uso abusivo de suplementos nutricionais. Porém, previamente à indicação de qualquer suplementação, faz-se necessária uma orientação dietética adequada. A ingestão da quantidade correta de calorias, carboidratos, proteínas, gorduras, vitaminas e minerais é a base fundamental para qualquer praticante de atividade física. E a suplementação primária deve ocorrer quando não se consegue suprir a necessidade diária dos nutrientes através da alimentação. Já foram descritos mais de duzentos e cinquenta tipos de suplementos alimentares. Os suplementos podem conter carboidratos, proteínas, gorduras, minerais, vitaminas, ervas, enzimas, substâncias do metabolismo intermediário (como aminoácidos) e vários tipos de extratos vegetais e animais. Este artigo é uma revisão sobre os suplementos nutricionais que apresentam embasamentos científicos consistentes: carboidratos, proteínas, BCAAs (Aminoácidos de Cadeia Ramificada) e creatina. O intuito é de prover um esclarecimento e orientar a população médica quanto à indicação, benefícios e riscos dessas substâncias.

Palavras Chave: Suplementos nutricionais, Proteínas, Carboidratos, Creatina, Aminoácidos de Cadeia Ramificada.

\section{ABSTRACT}

The promise of quick results in muscle mass, body definition, fat reduction and body weight is contributing to the abuse of nutritional supplements. However, prior to the prescription of any supplementation, it is necessary to adequate the nutrients intake from the diet. The intake of the right amount of calories, carbohydrates, proteins, fats, vitamins and minerals is the fundamental basis for any physical activity practitioner. And the primary supplementation should occur when you cannot meet the daily requirement of nutrients through food. There are over two hundred and fifty types of food supplements described. The supplements may contain carbohydrates, proteins, fats, minerals, vitamins, herbs, enzymes of the intermediary metabolism substances (such as amino acids) and various types of animal and plant extracts. This article is a review of the nutritional supplements which have consistent scientific studies: carbohydrates, proteins, BCAAs (Branched Chain Amino Acids) and creatine. The aim is to provide clarification and guide the medical community about the indications, benefits and risks of these substances.

Key Words: Dietary Supplements, Proteins, Carbohydrates, Creatine, Amino-acids-Branched-Chain 


\section{INTRODUÇÃO}

O número de usuários de suplementos nutricionais está aumentando exponencialmente no Brasil e nos demais países, por diversos motivos como: benefícios para a saúde em geral, busca incessante pelo "corpo perfeito", melhora no condicionamento físico e ganho no desempenho dos atletas de elite. 1-3

A promessa de resultados rápidos e milagrosos no ganho de massa muscular, definição corporal, redução de gordura e peso corporal vem contribuindo para o uso abusivo dessas substâncias 4 . No Brasil, o Ministério da Saúde e a ANVISA, definem suplementos nutricionais como: alimentos compostos exclusivamente por nutrientes vitamínicos e/ou minerais, que servem para complementar a dieta diária de uma pessoa saudável 5. Por essa definição ser extremamente genérica, são frequentes os lançamentos de novos supostos suplementos nutricionais sem uma regulamentação adequada e controle rígido de benefícios e malefícios dessas substâncias.

Em pesquisa recente feita com cento e cinquenta frequentadores de uma loja de suplementos foi visto que: $44 \%$ dos entrevistados faziam uso de algum tipo de suplemento por iniciativa própria, $15 \%$ por indicação de um professor de educação física, 12\% por indicação de um amigo, 16\% por demais indicações e apenas $9 \%$ por indicação de um médico 3. Muitos profissionais da área da saúde permanecem incertos na recomendação de suplementos nutricionais. 0 principal motivo para isso é a falta de formação adequada para realizar a orientação correta e fornecer informações a respeito da real indicação, da segurança e dos benefícios dos mesmos.

Este artigo é uma revisão sobre os suplementos nutricionais que apresentam embasamentos científicos consistentes. $O$ intuito é de prover um esclarecimento e orientar a população médica quanto à indicação, benefícios e riscos dessas substâncias. Na segunda parte desta revisão o foco serão os indivíduos idosos, pelas particularidades inerentes a esta parcela da população.

\section{RECOMENDAÇÃO NUTRICIONAL}

Previamente à indicação de qualquer suplementação, faz-se necessária uma orientação dietética adequada. A ingestão da quantidade correta de calorias, carboidratos, proteínas, gorduras, vitaminas e minerais é a base fundamental para qualquer praticante de atividade física. Para isso, a ISSN (International Society of Sports and Nutrition) publica recomendações periódicas sobre a quantidade adequada dos macro e micronutrientes na alimentação, que deve ser individualizada, dependendo da carga de atividade física de cada indivíduo. A última publicação da ISSN encontra-se na tabela 14,6 . Algumas particularidades em relação aos idosos e dietas hipocalóricas serão descritas posteriormente.

Tabela 1 - Recomendações da ISSN da quantidade da ingestão de macronutrientes em relação à atividade física

\begin{tabular}{|c|c|c|c|}
\hline $\begin{array}{l}\text { Quantidade/ } \\
\text { Atividade } \\
\text { física }\end{array}$ & $\begin{array}{l}3 x / \text { seman } \\
a \\
30-40 \\
\text { minutos }\end{array}$ & $\begin{array}{l}5- \\
6 x / \text { seman } \\
a \\
120-180 \\
\text { minutos }\end{array}$ & $\begin{array}{l}5- \\
6 x / \text { sema } \\
\text { na } \\
180-360 \\
\text { minutos }\end{array}$ \\
\hline Calorias & $\begin{array}{l}25 \text { a } 35 \\
\mathrm{kcal} / \mathrm{kg} / \mathrm{di} \\
a\end{array}$ & $\begin{array}{lll}50 & \text { a } 80 \\
\mathrm{kcal} / \mathrm{kg} / \mathrm{dia}\end{array}$ & $\begin{array}{l}50 \text { a } 80 \\
\mathrm{kcal} / \mathrm{kg} / \mathrm{di} \\
\text { a }\end{array}$ \\
\hline Carboidratos & $\begin{array}{l}45-55 \quad \% \\
\text { dieta } \\
(3 \quad \text { a } 5 \\
\text { g/kg/dia) }\end{array}$ & $\begin{array}{l}55-65 \% \\
\text { dieta } \\
\begin{array}{ll}(5 \quad \text { a } & \\
\text { g/kg/dia) }\end{array}\end{array}$ & $\begin{array}{l}8 \text { a } 10 \\
\text { g/kg/dia }\end{array}$ \\
\hline Proteínas & $\begin{array}{l}0,8 \text { a } 1 \\
\mathrm{~g} / \mathrm{kg} / \mathrm{dia}\end{array}$ & $\begin{array}{l}1 \text { a } 1,5 \\
\mathrm{~g} / \mathrm{kg} / \mathrm{dia}\end{array}$ & $\begin{array}{l}1,5 \text { a } 2 \\
\text { g/kg/dia }\end{array}$ \\
\hline Gordura & $30 \%$ dieta & $30 \%$ dieta & $30 \%$ dieta \\
\hline
\end{tabular}

Legenda: adaptada conforme recomendações da ISSN de $2010^{6}$

\section{INDICAÇÕES DA SUPLEMENTAÇÃO NUTRICIONAL}

A suplementação primária deve ocorrer quando não se consegue suprir a necessidade diária dos nutrientes através da alimentação. Uma segunda indicação da suplementação relaciona-se com a sua praticidade, como: falta de tempo em se fazer uma refeição adequada, antes ou após o exercício ou mesmo durante um treino de longa duração 6,7.

0 efeito ergogênico de algumas substâncias também é um objetivo da suplementação. O termo ergogênico é derivado da palavra grega "ergon" (trabalho) e "gennan" (produzir). Portanto, recursos ergogênicos são definidos de uma forma abrangente como: qualquer estratégia capaz de maximizar a capacidade de trabalho. Particularmente, existe um grande interesse sobre os suplementos nutricionais que, supostamente, potencializam o resultado obtido com algum tipo de atividade física. O processo de hipertrofia da musculatura esquelética em resposta ao treinamento de força é o mais estudado e o mais relacionado à ergogenia da suplementação 4,8.

Já foram descritos mais de duzentos e cinquenta tipos de suplementos alimentares. Os suplementos podem conter carboidratos, proteínas, gorduras, minerais, vitaminas, ervas, enzimas, 
substâncias do metabolismo intermediário (como aminoácidos) e vários tipos de extratos vegetais e animais. Eles podem ser classificados como suplementos de conveniência (por exemplo: barras energéticas e bebidas com carboidratos e minerais), os quais são formulados para fornecer uma quantidade adequada de calorias e substâncias necessárias; suplementos que agem no ganho ou perda de peso e suplementos que agem melhorando o desempenho 6 .

$\mathrm{Na}$ sequência desta revisão serão descritos apenas nutrientes e substâncias que apresentam embasamento científico que comprovam a sua eficácia, e que sejam legalizados para o uso em geral e no esporte. As indicações e contra-indicações estão resumidas na tabela 2 .

Tabela 2 - Resumo das indicações e contraindicações dos suplementos

\begin{tabular}{|c|c|c|}
\hline Suplemento & Indicações & $\begin{array}{l}\text { Contra- } \\
\text { Indicações }\end{array}$ \\
\hline Carboidrato & $\begin{array}{l}\text { Exercícios longa } \\
\text { duração }\end{array}$ & Diabéticos \\
\hline $\begin{array}{l}\text { Proteínas } \\
\text { (Whey- } \\
\text { Protein) }\end{array}$ & $\begin{array}{l}\text { Baixa ingestão } \\
\text { através da dieta }\end{array}$ & $\begin{array}{l}\text { Alérgicos à } \\
\text { proteína do leite; } \\
\text { Doentes Renais } \\
\text { Crônicos; } \\
\text { Hepatopatas }\end{array}$ \\
\hline BCAAs & $\begin{array}{l}\text { Ganho massa } \\
\text { muscular, final } \\
\text { provas longas e } \\
\text { potencial efeito } \\
\text { anti-inflamatório }\end{array}$ & $\begin{array}{l}\text { Doentes Renais } \\
\text { Crônicos; } \\
\text { Hepatopatas }\end{array}$ \\
\hline Creatina & $\begin{array}{l}\text { Ganho de } \\
\text { massa e força } \\
\text { muscular } \\
\text { Reabilitação } \\
\text { física }\end{array}$ & - \\
\hline
\end{tabular}

\section{CARBOIDRATOS}

Os primeiros estudos com suplementação de carboidratos (CHO) foram feitos em 1986 pela indústria do Gatorade ${ }^{\circledR}$. Foi demonstrado que, após três horas de atividade física, a suplementação de $\mathrm{CHO}$ pouparia - glicogênio muscular nos últimos estágios do exercício. Porém, diversos estudos posteriores não conseguiram repetir os mesmos resultados 9-11.

Outro trabalho, do ano de 2008, demonstrou em condições experimentais, com nove ciclistas, a ocorrência do efeito placebo com a suplementação de $\mathrm{CHO}$. No momento em que os ciclistas utilizaram carboidrato e placebo cegamente, isto é, quando não sabiam o que estavam utilizando, não houve diferença no tempo para a fadiga com a atividade física intensa. Porém, quando os ciclistas sabiam que estavam utilizando $\mathrm{CHO}$ houve diferença significativa em relação ao placebo no tempo para a fadiga. Alguns ciclistas conseguiram permanecer até meia hora a mais na bicicleta até atingir a exaustão 12 .

Estudos recentes têm demonstrado algumas áreas do Sistema Nervoso Central (SNC) que podem estar relacionadas ao aumento do desempenho com o uso de $\mathrm{CHO}$ 13-15. Essas áreas são ativadas por receptores na mucosa oral após bochecho com solução de glicose e maltodextrina. Em um estudo feito com oito ciclistas, sete tiveram um tempo de execução reduzido para completar a quilometragem proposta em relação ao placebo $(60,4 \pm 3,7$ minutos - quando fizeram bochecho com solução de glicose e 61,6 6 3,8 minutos - quando bochecho com placebo) com $p=0,007$. Da mesma forma, os mesmos sete ciclistas também completaram em menos tempo a quilometragem quando fizeram bochecho com maltodextrina $(62,6 \pm 4,7$ minutos contra placebo $64,6 \pm 4,9$ minutos com $p=0,012$ ). Neste estudo foram feitos exames de Ressonância Magnética Nuclear do SNC e foi demonstrado que existem áreas de ativação com a solução de glicose e maltodextrina e talvez essas áreas estejam relacionadas com $\mathrm{o}$ aumento do desempenho 16.

Uma revisão sistemática de 2013 incluindo 17 estudos envolvendo a suplementação de $\mathrm{CHO}$ e tendo como base a resposta na melhora ou não no desempenho em ciclistas, concluiu que para atividades acima de setenta minutos de execução, a suplementação com $\mathrm{CHO}$ melhora o desempenho em torno de 1 a $13 \% 17$.

Outra revisão recente com 61 estudos $(n=679)$ evidenciou que $82 \%$ dos trabalhos demonstraram benefícios no desempenho. Os mecanismos relacionados a esse desfecho, que não são mutuamente exclusivos, dependem da duração do exercício. Em atividades de curta duração (menos que 1 hora), os receptores orais de $\mathrm{CHO}$ quando estimulados provocam realmente uma melhora no desempenho por ativarem áreas do prazer e da recompensa no SNC. Em exercícios de longa duração (mais que 2 horas), o mecanismo primário é pela maior taxa de oxidação de $\mathrm{CHO}$ gerando energia 18.

Segundo as recomendações da ISSN, a suplementação com $\mathrm{CHO}$ é altamente eficiente para exercícios aeróbios de longa duração. A ingestão alimentar prévia a atividade física, se feita mais do que uma hora antes do exercício físico, deve ser baseada em alimentos com baixo índice glicêmico. Caso seja feita em menos de uma hora para o exercício físico, deve ser baseada em alimentos com alto índice glicêmico. A quantidade recomendada de carboidratos deve ser de um grama por quilo de peso 6 .

Durante a atividade física, a recomendação é de suplementação em caso de exercício por mais de sessenta minutos. Nesse caso, a ingestão de $\mathrm{CHO}$ deve ocorrer a cada quinze a vinte minutos após a primeira hora e em torno de seis a oito gramas de $\mathrm{CHO}$. Após a 
atividade física é essencial a ingestão de $\mathrm{CHO}$ para a correta reposição dos estoques de glicogênios muscular e hepático. Estes levam de doze a quarenta e oito horas para serem ressintetizados. Assim, a preferência é pela ingestão de $\mathrm{CHO}$ de alto índice glicêmico - 0,7 gramas por quilo de peso - logo após o término do exercício. Posteriormente, em trinta a quarenta minutos, deve ser feita uma refeição mista (contendo $\mathrm{CHO}$, proteínas e gorduras) 4,6.

\section{PROTEÍNAS}

Existe bastante controvérsia na literatura quanto à suplementação de proteínas para praticantes de atividade física 19-22. Entretanto, nas últimas décadas, os estudos indicam que indivíduos que praticam atividade física intensa necessitam, para a manutenção de um balanço proteico positivo, de um aporte de proteína duas vezes maior do que a quantidade indicada pela IDR (Ingestão Diária Recomendada), que é de 0,8 a 1 grama por quilo de peso por dia 4,6,23-25.

A necessidade máxima de proteína diária em indivíduos que praticam atividade física intensa é de dois gramas por quilo de peso por dia. Uma quantidade acima desse valor pode gerar, inclusive, uma piora no desempenho, como demonstrado por Porttmans e col. 26. Para um melhor aproveitamento, a quantidade de proteína ingerida deve ser equilibrada entre as refeições. Esse fracionamento é importante, pois as vilosidades intestinais possuem uma capacidade de absorção de aminoácidos diferenciada, dependendo da composição química dos mesmos. Essa absorção ocorre por diferentes sistemas de transporte, e todos esses sistemas possuem limites de saturação 27,28.

Os principais benefícios da utilização de um suplemento proteico são: 6

Repor a quantidade diária necessária de proteínas quando esta não é obtida através da alimentação;

- Maior facilidade de digestão, importante principalmente para idosos com dificuldades de mastigação, deglutição e digestão;

- Menor aporte de gorduras, visto que alimentos ricos em proteínas apresentam taxas de gordura mais elevadas do que suplementos proteicos isolados;

-Maior velocidade de absorção, característica extremamente importante para a síntese de proteínas musculares.

Existem três tipos principais de suplementos proteicos, o mais utilizado é o Whey-Protein (proteína do soro do leite, rico em aminoácidos essenciais). Cada dosagem contém cerca de 20 a 25 gramas de proteína, dependendo da marca. Os outros tipos são a Caseína, que também é derivada do leite, e a proteína da soja. Tang e col. demonstraram que a taxa de síntese proteica muscular foi mais elevada após atividade física no grupo que utilizou o Whey-Protein (122\% maior comparada ao grupo que utilizou a caseína e $32 \%$ maior em relação ao grupo que utilizou a proteína derivada da soja) 29. Porém, há outros estudos que evidenciaram uma boa eficácia na síntese protéica muscular com os três tipos de proteínas 30,31. A principal diferença entre elas está na velocidade de absorção.

A síntese protéica muscular apresenta um turnover constante. O aumento na degradação proteica após atividade física ocorre desde o início da atividade até seis horas após o exercício, com queda posterior nas próximas quarenta e oito horas. A síntese proteica vai ocorrer, em indivíduos jovens, principalmente nas três primeiras horas após uma atividade física (período denominado de "janela anabólica"). Essa síntese vai permanecer em um platô por vinte e quatro horas e ocorrerá em menor intensidade até completar quarenta e oito horas. Dessa maneira, uma das explicações para um maior benefício de uma proteína de rápida absorção seria sua ação na chamada "janela anabólica" 30-32.

Alguns estudos divergem em relação ao momento correto do uso de uma suplementação proteica, se antes ou após um treino de resistência 3336. Entretanto, foi demonstrado em um trabalho clássico, feito com trinta homens que utilizaram por doze semanas uma suplementação com dez gramas de proteína antes ou após treino, que o grupo que utilizou o suplemento após a atividade física apresentou um ganho maior na área média de fibra muscular vista por DXA (Absortometria por Dupla Emissão de RX), na área seccional de quadríceps visto na RNM (Ressonância Magnética Nuclear) e também um ganho maior de força verificado em aparelhos de resistência 37.

O uso de proteínas em exercícios de endurance (resistência) também apresenta benefícios demonstrados em alguns estudos na literatura 38-41. Um trabalho recente 42 comparou o uso de $\mathrm{CHO}$, associado a uma ingestão de 1,2 gramas de proteína por quilo de peso por dia, e $\mathrm{CHO}$ com o dobro da quantidade de proteína por quilo de peso por dia, num período de 14 dias. No décimo quinto dia os atletas foram submetidos a sessenta minutos de atividade física aeróbica a 70\% do VO2 máximo. Após esse período, os mesmos foram submetidos a exercícios a $90 \%$ do VO2 máximo até a fadiga. Houve uma diferença significativa em relação ao nível de insulinemia aos cento e oitenta minutos, mais elevado no grupo que estava com uma suplementação maior de proteínas. Este nível mais elevado de insulina pode ter contribuído para uma melhora na recuperação do glicogênio muscular visualizada neste grupo após o exercício. Outra diferença significativa, foi a melhora da biogênese mitocondrial, evidenciada por um aumento na expressão do PGC $1 \alpha$ (fator de transcrição 
responsável pelo estímulo da biogênese mitocondrial gerando uma melhora na capacidade aeróbica).

O estímulo mecânico da atividade física de resistência, o hormônio de crescimento, a insulina e os aminoácidos geram um aumento na síntese proteica muscular através de diversas vias com estímulo final na via de sinalização mTOR (mammalian target of rapamycin) 32. O treinamento de resistência, quando feito com um superávit de proteínas, consegue aumentar a síntese proteica muscular em torno de 40 a $100 \%$ 43-46. Na literatura existem mais de três mil artigos relacionando a suplementação de proteínas com um aumento no desempenho e no ganho de massa muscular em atividade física de resistência. Uma meta-análise recente que avaliou vinte e dois estudos com 680 indivíduos entre 19 e 72 anos mostrou que a suplementação com proteínas deveria ser acima de 1,2 gramas por quilo de peso por dia por, no mínimo seis e máximo vinte e quatro semanas, associada a exercício resistido. As diferenças estatisticamente significativas foram: um ganho médio na taxa isenta de gordura de $38 \%$ no grupo que utilizou uma suplementação de proteína em relação ao placebo e um ganho de 33\% na avaliação do desempenho do exercício de repetição máxima vista no aparelho de leg-press 47.

Em resumo: a última recomendação feita pela ISSN e pelo Anual Congress ECSS 48 é de orientar a suplementação com vinte gramas de Whey-Protein após o treino se necessário; isto é, caso a ingestão alimentar através de alimentos não seja suficiente para suprir a demanda necessária diária de proteína, sempre relacionando com o peso e a quantidade de exercício físico semanal. Caso o treino seja feito logo antes ao período de sono, pode ser feito o uso de quarenta gramas de caseína ou um copo de leite de $200 \mathrm{ml}$. Existe a sugestão para que, em dietas hipocalóricas que objetivam perda ponderal, idealmente a ingestão de proteína deveria ser de 35\% do valor calórico total diário, evitando, assim, uma perda de massa magra 6,49 .

\section{BCAAs (Aminoácidos de Cadeia Ramificada)}

Os BCAAs são aminoácidos (aa) essenciais, isto é, aminoácidos que não são produzidos no organismo e são adquiridos apenas através da alimentação. A diferença em relação aos demais aa essenciais é que estes são metabolizados no músculo. São eles: a leucina, a valina e a isoleucina. Diversos estudos demonstraram que os BCAAs agem na síntese proteica por estímulo de vias que irão levar a uma ativação final da via da mTOR. A leucina, entre os três, parece ser o mais eficaz 50-52.

Um estudo com dezesseis pacientes demonstrou que o grupo que utilizou BCAA, em relação ao grupo controle, após duas horas de uma sessão de exercício resistido, teve um aumento de $145 \%$ na fração de síntese proteica muscular vista por biópsia muscular,enquanto isso, o grupo controle, teve um aumento de $41 \%$. O estímulo da via mTor também foi estatisticamente diferente em relação aos dois grupos 50. Outro trabalho com leucina associado a $\mathrm{CHO}$ e proteína, demonstrou um beneficio no ganho de massa muscular em relação ao grupo que utilizou apenas $\mathrm{CHO}$ comparado a outro grupo que utilizou $\mathrm{CHO}$ e proteína 52.

Outro beneficio do uso dos BCAAs vem sendo visualizado em momentos finais de provas longas, como maratona ou iron-man. O motivo seria a participação destes no ciclo de Cori (reação anaplerótica), em que ocorre a formação de glicose através dos aa. Lancha e col. demonstraram que ratos que utilizaram BCAAs, quando levados à exaustão, obtiveram uma maior resistência à fadiga, possivelmente pelo aumento na taxa de glicogênio hepático em relação ao grupo placebo 53.

Os BCAAs também estão sendo estudados por possuírem potentes efeitos anti-inflamatórios. Eles podem auxiliar em um menor tempo de recuperação de lesões e em uma recuperação muscular mais precoce após um exercício de esforço intenso. Esse mecanismo está associado à inibição da produção de TNF- $\alpha$ (fator de necrose tumoral-alfa), IL-6 (interleucina-6), NF-K $\beta$ (fator nuclear $\kappa \beta$ ) e ROS (espécies reativas de oxigênio) 49.

Em jovens, um grama de leucina parece ser suficiente para agir como um gatilho para a síntese proteica muscular. As últimas recomendações são: orientar o uso de 8,6 a 10 gramas de aa essenciais após o treino. Os Whey-Proteins existentes no mercado já apresentam quantidades suficientes de BCAAs, não sendo necessário um uso suplementar isolado destas substâncias 6,54,55.

CREATINA

A creatina é uma amina de ocorrência natural sintetizada endogenamente pelo fígado, rins e pâncreas, a partir dos aminoácidos glicina, arginina e metionina. Esta produção endógena é geralmente de um grama por dia. Através da dieta ingerimos também em torno de um grama por dia, principalmente pelo consumo de carne vermelha e peixes. A creatina encontra-se $95 \%$ dentro do tecido muscular, sendo 30 a $40 \%$ em sua forma livre e 60 a $70 \%$ na forma fosforilada. A taxa de degradação da creatina é de dois gramas por dia. Essa degradação ocorre espontaneamente, por uma reação não enzimática, tanto a creatina, quanto a fosfocreatina, são transformadas em creatinina 56,57.

Definida como o suplemento mais eficiente para hipertrofia muscular e considerado segura quando utilizada nas dosagens recomendadas. Apresenta-se em maiores níveis nas fibras musculares tipo Ila e Ilb, 
as quais estão mais relacionadas à contração rápida e à força muscular. A creatina age contribuindo na geração de energia. Ela abastece o sistema creatino-fosfato, que é uma importante fonte de liberação rápida e precoce de energia. Ela age, também, na redução da formação do lactato. Apresenta maior efetividade em atividades de sprints, melhorando o desempenho em atividades de combate, modalidades coletivas e provas de curta duração (menores que trinta segundos). Entretanto, em provas de longa duração, e provas que dependam de uma massa corporal mais baixa, a creatina pode até piorar o desempenho 6,57-63.

Uma meta-análise demonstrou um aumento na massa magra e no ganho de força muscular em três semanas de treino, com atividades duas vezes na semana 62. Em revisão recente, feita por Gualano e col. 60, foi concluído que os ganhos de força e massa magra advindos da suplementação de creatina são consequências dos aumentos de retenção hídrica, expressão gênica e eficiência da tradução de proteínas relacionadas à hipertrofia, além da proliferação e ativação de células satélites. À luz da presente literatura, ainda não se pode afirmar com clareza se essas adaptações são ocasionadas por efeitos diretos da suplementação de creatina ou se são mediadas pelo aumento no volume de treinamento. Contudo, os efeitos da suplementação de creatina na promoção de ganho de massa magra e força são contundentes. Segundo diretriz publicada pela Sociedade Brasileira de Medicina do Esporte em 2003 64, não havia evidências científicas suficientes que embasassem o emprego da suplementação de creatina com fins ergogênicos. Diante da consistência dos achados vistos nesta revisão, acreditamos que as recomendações sobre esse suplemento precisam ser revisadas e atualizadas.

$\mathrm{O}$ aumento da força muscular foi demonstrada em estudo com 18 jovens com o uso de creatina, na dose de trinta gramas por dia por uma semana e após cinco gramas por dia por mais sete semanas, associada a treinamento de resistência. O grupo que usou creatina aumentou o peso corporal, com ganho de massa magra, massa isenta de gordura e força muscular $(p<0,05) 65$.

Em uma meta-análise de sessenta e sete estudos, quarenta e três mostraram que a creatina aumenta a massa corporal total ou a massa magra 63 . Entretanto, o seu efeito no balanço proteico não ocorre de maneira aguda e sim por uma hipertrofia muscular em longo prazo. A creatina, como já sugerido, permite um aumento no volume de treinamento, ou seja, a sua suplementação permite um número maior de séries a serem executadas. A creatina também age estimulando a proliferação e diferenciação das células satélites, que são importantes para o processo de hiperplasia e hipertrofia muscular 60,64,65.

Outros prováveis benefícios da creatina, que estão em estudo, são: reabilitação física (acelerando o ganho de massa muscular), em doenças neuromusculares (por modificar o potencial de ação da membrana celular - gerando um efeito mais rápido na sinalização para a produção da contração muscular) e efeitos na cognição (demonstrando boas respostas nos tratamentos de doenças de Alzheimer, Parkinson e depressão) 66,67.

O maior receio quanto ao uso da creatina é o seu efeito na função renal. O que se tem comprovado é que a creatina realmente está relacionada a um ganho de peso e retenção hídrica. Porém, esta retenção ocorre dentro dos músculos, fato que está associado a um favorecimento da tradução de proteínas contráteis, devido à alteração da osmolaridade intracelular. Existem diversos trabalhos até o momento que avaliaram se a creatina seria prejudicial para a função renal. Porém, nenhum estudo até o momento evidenciou nenhum prejuízo,corroborado por uma revisão 68-70.

Devido a degradação espontanea da creatina em creatinina, o clearance de creatinina deixa de ser um bom marcador de função renal em casos de suplementação. Este clearance pode estar elevado e não representar uma piora real da função renal. Um estudo feito por Gualano e col. avaliou a função renal através da dosagem da cistatina $C$, que é uma proteína de baixo peso molecular totalmente filtrada $e$ reabsorvida, e que não sofre influência da alimentação e da massa muscular. Neste estudo, a creatina foi usada em altas doses (dez gramas por dia, por três meses) e foi comparada com placebo. Não houve diferença entre os grupos em relação aos parâmetros de função renal analisados 71 .

Não foi demonstrado, também, deterioração da função renal com o uso de creatina em paciente com rim único 72 , assim como nos pacientes com diabetes mellitus tipo 273 . Neste estudo foi feito o uso de cinco gramas ao dia de creatina, por doze semanas, num grupo de treze pacientes com diabetes mellitus tipo 2. Destes, cinco já apresentavam algum grau de insuficiência renal. Foi feita a comparação com um grupo placebo de doze pacientes, sendo cinco destes também com algum grau de insuficiência renal. A avaliação da função renal foi determinada pelo exame de clearance de crômio EDTA. Os resultados não mostraram diferença entre os grupos. Em alguns pacientes do grupo intervenção houve melhora do controle glicêmico, com diminuição de até um ponto na hemoglobina glicada $(p=0,004)$. Esta melhora da hemoglobina glicada provavelmente ocorreu porque a creatina aumenta a transcrição do transportador GLUT 4 para a membrana celular. Desse modo, a melhora da função renal pode ser explicada pela melhora do controle glicêmico.

A última recomendação da ISSN sugere que a dosagem de creatina que, aparentemente, atinge efeitos mais rápidos, deve ser de 0,3 gramas por quilo 
de peso por dia, por pelo menos três dias, seguida de três a cinco gramas por dia para manter os estoques necessários. A ingestão de doses menores, como dois a quatro gramas por dia, por um período de três a quatro semanas, leva a um aumento no estoque de creatina intramuscular, porém os efeitos no desempenho com essa dosagem são menos estudados 6 .

Um resumo dos principais sítios de ação dos suplementos do artigo está ilustrado na figura 1.

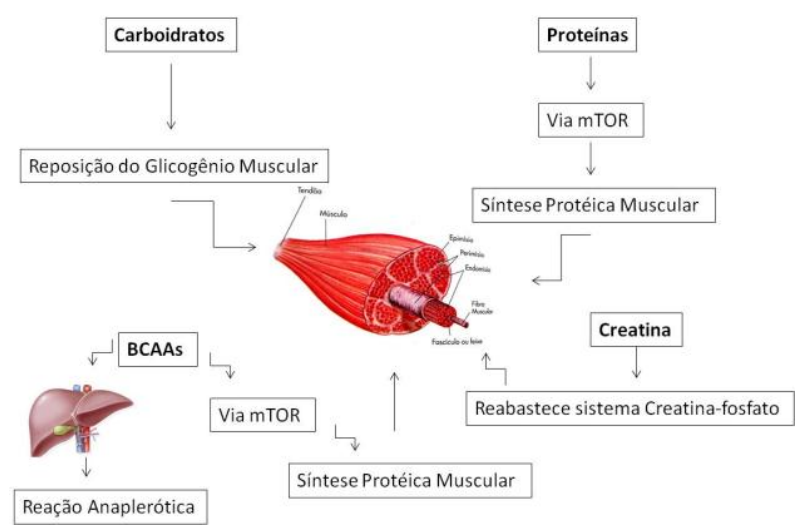

Figura 1- Principais sítios de ação dos suplementos

\section{IDOSOS}

A suplementação nutricional em idosos é um capítulo a parte, devido às diversas diferenças e peculiaridades no metabolismo e na resposta aos suplementos nesta faixa etária. O uso de substâncias que auxiliem na perda de massa magra que acontece nesta população está sendo cada vez mais estudado e utilizado. A sarcopenia (perda de massa magra) é uma alteração extremamente prevalente em idosos. Entre 60 a 70 anos, a prevalência é de $5 \%$ a $13 \%$, e, acima dos 80 anos, em torno de $11 \%$ a $50 \%$. Já está bem estabelecido, que acima dos 50 anos, existe uma redução da massa muscular em torno de $1 \%$ a $2 \%$ ao ano. Além da perda de massa muscular, ocorre uma redução da força muscular, o que acarreta redução de mobilidade, dificuldade na realização de atividades diárias e, finalmente, redução na qualidade de vida. Entre 50 a 60 anos a perda da força muscular chega a $1,5 \%$ ao ano. Após os 60 anos, essa perda passa a ser em torno de $3 \%$ ao ano. Os motivos da sarcopenia são diversos: fatores nutricionais, hormonais, inflamatórios, perda de neurônio motor e redução de fibras musculares tipo II, entre outros 74-76.

O diagnóstico da sarcopenia deve ser feito pela demonstração de redução da massa muscular, associada sempre à alteração funcional ou à redução de força muscular. O último consenso europeu de sarcopenia define como pré-sarcopenia quando: existe apenas redução da massa muscular; sarcopenia quando presente a redução da massa muscular associada à redução da força muscular ou do desempenho em testes funcionais e sarcopenia severa quando as três características estão presentes 77 .

Diversos estudos estão em andamento em relação ao tratamento da sarcopenia. Os mais pesquisados são: intervenção com exercícios físicos aeróbicos e de resistência, terapias hormonais (uso de testosterona, estrogênio e hormônio de crescimento), vitamina $D$, uso de inibidores da ECA e novos potenciais terapêuticos, como antagonistas da miostatina, agonistas PPAR $\delta$ (receptores ativados por proliferadores de peroxissoma delta) e AICAR (5aminoimidazole-4-carboxamida-1- $\beta$-D-ribofuranoside ativador AMPK). Além dessas intervenções, existem na literatura inúmeros trabalhos com o uso de suplementos nutricionais que são o foco dessa revisão 78,79 .

Algumas diferenças na síntese proteica muscular e na resposta à atividade física são importantes fatores a serem considerados na indicação da orientação alimentar mais adequada, caso seja necessário uma suplementação nutricional nesta faixa etária. Existe um atraso na síntese proteica dos idosos, quando comparados com indivíduos jovens, após um estímulo anabólico 80,82. A chamada "janela anabólica" nesta população ocorre de três a seis horas após o estimulo.

As causas desse atraso também foram estudadas por esse mesmo estudo através de biópsia muscular. As diferenças moleculares não foram relacionadas à via da mTOR, ao 4E-BP1, S6K1 e eFF2 que são fatores de transcrição relacionados à síntese proteica muscular, e sim no fator ERK1/2 (fator de transcrição também relacionado à síntese proteica muscular da via da MAPKinase): este fator, na primeira hora após exercício, estava elevado nos jovens, mas não nos idosos. Outra diferença foi no fator AMPKa (fator de transcrição relacionado à redução da síntese proteica muscular): nos idosos não houve uma redução da sua expressão nas primeiras três horas após o exercício, diferentemente dos jovens, nos quais foram observados níveis reduzidos 83 .

Uma modalidade de exercício físico chamado BFR (blood flow restriction- restrição do fluxo sanguineo) está sendo estudada nesta população. Esta modalidade é interessante para ser utilizada justamente em indivíduos com lesões ou que apresentam dificuldades em utilizar exercícios de resistência com cargas elevadas. Ela gera um aumento da massa e força muscular, porém com apenas $20 \%$ da carga máxima em que uma pessoa consegue fazer uma repetição (conceito denominado $1 \mathrm{RM}$ ). Através de bandas elásticas ou cuffs ocorre uma oclusão do sistema venoso e, com isso, um estímulo maior ao metabolismo anaeróbio. Esse mecanismo vai gerar 
uma fadiga mais precoce das fibras lentas (tipo I) e uma utilização mais precoce das fibras tipo II (mais relacionadas à hipertrofia e força muscular) 84-86. Fry e col. demonstraram que essa modalidade de atividade física leva a um estímulo maior da via mTOR e redução da miostatina (proteína associada à inibição do crescimento muscular). Também foi demonstrado que, após quatro séries de 15 a 20 repetições, a $20 \%$ da 1 RM, em nove idosos, houve um aumento de $56 \%$ na síntese proteica muscular após 3 horas do exercício resistido com a BFR 87.

Além do atraso na síntese proteica muscular após o estímulo anabólico, existe também, na população idosa, um mecanismo chamado de "resistência anabólica". Isso significa que é necessário uma quantidade maior de proteínas para ser atingida a mesma síntese proteica dos jovens 88,90. Um estudo demonstrou que após cinco gramas de proteína, os jovens já iniciam a síntese proteica muscular e atingem um platô após vinte gramas. Já em idosos, esse nível de proteína deve ser mais elevado: em torno de dez gramas para início e trinta gramas para o platô 7. 0 exercício de resistência nesta população também gera uma menor resposta na síntese muscular. As possíveis explicações para isso são: a alteração na função endotelial (levando menos substâncias responsáveis pela síntese proteica para a musculatura esquelética) e a alteração na sinalização da via anabólica (por um possível baixo grau de inflamação que ocorre nesta população) 91,92.

Em resumo, pelas características distintas da população idosa em relação aos jovens, a ingestão de ideal de proteínas para manter a massa muscular ao longo dos anos é de 30 gramas em cada refeição (sendo 10 gramas de aa essenciais de preferência), conforme demonstrado por Paddon-Jones e col. 93. Caso não seja atingida essa quantidade através da ingestão alimentar, deve ser feito o uso da suplementação para se conseguir a quantidade ideal para essa faixa etária, prevenindo assim a sarcopenia 6 .

Alguns estudos demonstram que pode ser possível vencer a "resistência anabólica" que existe nesta população através do uso de leucina em doses mais elevadas. Nos jovens é conhecido que um grama de leucina já estimula a síntese protéica muscular. Em idosos, este estímulo ocorreria a partir de dois a três gramas por dia. Existem no mercado suplementos mais específicos para idosos que contêm essa quantidade mais elevada de leucina por dosagem 94.

O benefício do uso de creatina na população idosa foi demonstrado por diversos trabalhos que demonstraram ganho de massa magra e, ainda mais importante, no ganho de função, desempenho e qualidade de vida 95,98. O uso de 0,3 gramas por quilo de peso por dia de creatina para homens, quanto para mulheres acima de sessenta anos, melhorou todos os parâmetros acima relacionados 99,100. Um estudo com trinta homens de $70 \pm 1,4$ anos, utilizando por dez semanas creatina na dose de 0,3 gramas por quilo de peso por dia, por cinco dias, e após 0,07 gramas por quilo de peso por dia, associado a treino resistido, demonstrou melhora em ganho de $3,3 \mathrm{~kg}$ de massa magra, contra $1,3 \mathrm{~kg}$ no grupo placebo $(p<0,05)$; aumento na carga máxima utilizada no aparelho de legpress $(50,1 \mathrm{~kg}$, contra $31,3 \mathrm{~kg}$ no grupo placebo, com $p<0,05)$ e também um aumento no número de repetições do leg-press (47 contra 32 do grupo placebo, com $\mathrm{p}<0,05)$ demonstrando assim uma melhora também no exercício de endurance 95.

Em idosos frágeis, a creatina a dose de 5 gramas por dia, por seis meses, associado a exercícios de resistência, levou a um ganho de massa magra e melhora no desempenho de força muscular no grupo da creatina em relação ao placebo 101 . Um artigo de revisão concluiu que: em idosos, o uso de creatina em baixas dosagens, apenas três vezes na semana, associado a exercícios de resistência leva a um aumento da massa e força muscular 102.

\section{CONCLUSÃO}

A suplementação nutricional é um tema ainda de muita controvérsia. Existe, por grande parte dos profissionais médicos, um receio muito grande quanto ao uso dessas substâncias; algumas vezes por falta de domínio do assunto, outras por não acreditarem nos benefícios desses produtos. Porém, deve ser de conhecimento de toda a população médica que existem trabalhos científicos muito bem elaborados sobre o tema.

O problema maior ainda enfrentado pela suplementação é a falta de controle sobre esses produtos. Como são considerados alimentos, e não medicamentos, a fiscalização não costuma ser rígida. Existem trabalhos que demonstram a adição de substâncias proibidas como esteróides 103 em suplementos, fato que, logicamente, não aparecem nos rótulos. Além da adição de substâncias ilícitas, verificase que as informações quanto às concentrações de substâncias nos rótulos dos produtos podem estar incorretas, podendo chegar até a $69 \%$ menos da concentração real de proteína do que está indicado no rótulo 104.

Os estudos na área da suplementação são limitados. Geralmente o número de casos avaliado é pequeno, as variáveis analisadas ocorrem após uma ingestão aguda de uma substância e não são vistos os efeitos cronicamente. Alguns trabalhos apresentam erros por não apresentarem um controle nutricional adequado durante o estudo, por técnicas de avaliação inadequadas e análises de apenas uma substância ou um gene envolvido, sendo que, diversas variáveis agem 
em conjunto para a obtenção de um resultado satisfatório 4.

A suplementação nutricional deve ser encarada como um algo a mais. Primeiramente, sempre deve ser avaliado se a base nutricional está adequada, se existe um treinamento adequado (com tempos corretos de repouso e treinos intercalados). Deve, também, ser dada atenção para o momento certo da suplementação e a real necessidade. Porém, a principal limitação ainda é a falta de incentivo para pesquisas científicas nessa área. Suplementos nutricionais são ignorados pela forte indústria farmacêutica por não se enquadrarem na categoria "medicamentos". Além disso, pairam sobre todos os suplementos alimentares as impressões de fraude e charlatanismo, sustentadas por um comércio crescente de produtos sem benefícios e sem respaldo científico. Certamente, isso contribui para que a investigação de suplementos alimentares como agentes ergogênicos e terapêuticos seja prejudicada. Neste sentido, ocorre a necessidade de maior incentivo visto que diversos trabalhos demonstram benefícios em diversas variáveis analisadas 4

\section{REFERÊNCIAS}

1-Maughan, R. J.; Depiesse, F.; Geyer H. The use of dietary supplements by athletes.Journal of Sports Sciences. Vol. 25. Núm. 1. p. S103-S113 2007

2-Williams, M. H. Dietary supplements and sports performance: amino acids. Journal of the International Society of Sports Nutrition. Norfolk. Vol. 2. Núm. 2. p. 63-67 2005

3-Rigon, T. V.; Rossi G. T. Quem e porque utilizam suplementos alimentares. Revista Brasileira de Nutricao Esportiva. Vol. 6. Num. 36. p. 420-426 2012

4-Souza Junior, T. P.; Gualano, B.; Prestes, J.; Ribeiro, S. M. I.; Aoki, M. S. Biomotricity round table - dietary supplements and muscular hypertrophy. Brazilian Journal Biomotricity, Vol. 4, Num. 4. p. 2272452010

5-Gerência-Geral de Alimentos - Ouvidoria/anvisa e dpdc/senacon Consumo e Saude - Suplemento Alimentar fique atento. Ano 5 n.30, março de 2013

6-Kreider, R. B.; Wilborn C. B; Taylor L.; Campbell B.; Almada A. L.,Collins R. e col. ISSN Exercise \& Sport Nutrition Review: Research \& Recommendations. Journal of the International Society of Sports Nutrition 7:7 2010

7-Dideriksen K.; Reitelseder S.; Holm L. Influence of Amino Acids, Dietary Protein, and Physical Activity on Muscle Mass Development in Humans. Nutrients Num. 5. p. 852-876 2013

8-Nogiec C. D., Kasif S. To Supplement or Not to Supplement: A Metabolic Network Framework for Human Nutritional Supplements. Metabolic Modeling of Nutritional Supplements. Num 82013

9-Coyle E. F.; Coggan A. R..;Hemmert M. K., Ivy J. L. Muscle glycogen utilization during prolonged strenuous exercise when fed carbohydrate. American Physiological Society. 0161-7567/86 1989

10-Burke L.M.; Hawley J.A.; Schabort E.J.; St Clair G.A.; Mujika I.; Noakes T.D.. Carbohydrate loading failed to improve $100-\mathrm{km}$ cycling performance in a placebo-controlled trial. J Appl Physiol Num. 88. pg. 1284-1290. 2000

11-Jeukendrup A.E.; Hopkins S.; Aragon-Vargas L.F.; Hulston C. No effect ofcarbohydrate feeding on $16 \mathrm{~km}$ cycling time trial performance. Eur J Appl Physiol Vol. 104. pg. 831-837 2008

12-Nassif C.; Ferreia A. P. A.; Gomes A. R.; Silva L.; Garcia E.; Marino

F. Double blind carbohydrate ingestion does not improve exercise duration in warm humid conditions. Journal of Science and Medicine in Sport. Num. 11 pg. 72-79 2008

13-Carter J.M.; Jeukendrup A.E; Jones D.A. The effect ofcarbohydrate mouth rinse on 1-h cycle time trial performance. Med Sci Sports Exerc Num. 36. pg. 2107-2111. 2004

14-Araujo I.E.; Kringelbach M.L.; Rolls E.T.; Hobden P. Representation of umami taste in the human brain. J Neurophysiol Num. 90. pg. 313319. 2003

15-Lambert E.V.; St Clair Gibson A.; Noakes T.D. Complex systems model of fatigue: integrative homoeostatic control of peripheral physiological systems during exercise in humans. Br J Sports Med Num. 39 pg. 52-62. 2005

16-Chambers E. S. ; Bridge M. W; Jones D. A. Carbohydrate sensing in the human mouth: effects on exercise performance and brain activity. J Physiol Num. 587.8 pg. 1779-1794. 2009

17-Colombani P. C.; Mannhart C.; Mettler S. Carbohydrates and exercise performance in nonfasted athletes: A systematic review of studies mimicking real-life. Nutrition Journal 12:16. 2013

18-Stellingwerff T, Cox GR. Systematic review: Carbohydrate supplementation on exercise performance or capacity of varying durations. Appl Physiol Nutr Metab.2014 Sep;39(9):998-1011

19-Lemon P.W.; Tarnopolsky M.A.; MacDougall J.D.; Atkinson S.A. Protein requirements and muscle mass/strength changes during intensive training in novice bodybuilders. J Appl Physiol Núm. 73(2) pg. 767-75 1992

20-Tarnopolsky M.A. Protein and physical performance. Curr Opin Clin Nutr Metab Care Núm. 2(6) pg. 533-7 1999

21-Kreider R.B.; Kleiner S.M. Protein supplements for athletes: need vs. convenience. Your Patient \& Fitness Núm 14(6) pg. 12-8 2000

22-Campbell B.; Kreider R.B.; Ziegenfuss T., La Bounty .; Roberts M.; Burke D.; et al. International Society of Sports Nutrition position stand: protein and exercise. J Int Soc Sports Nutr 4:8 2007

23-American Dietetic Association; Dietitians of Canada; American College of Sports Medicine, Rodriguez N. R.; Di marco N. M.; Langley $\mathrm{S}$. American college of sports medicine position stand. Nutrition and Athletic Performance. Medicine and Science in Sports Exercise Núm. 3, pg. 709-31 2009

24-Bilsborough S.; Mann N. A review of issues of dietary protein intake in humans. International Journal of Sport Nutrition and Exercise Metabolism, Núm. 2, pg. 129-152 2006

25-Brasil. Ministério da Saúde. Agência Nacional de Vigilância Sanitária. Resolução RDC no 269, de 22 de setembro de 2005. O "REGULAMENTO TÉCNICO SOBRE A INGESTÃO DIÁRIA RECOMENDADA (IDR) DE PROTEÍNA, VITAMINAS E MINERAIS". ANVISA - Agência Nacional de Vigilância Sanitária, de 23 de setembro de 2005

26-Porttmans J. R.; Carpentier A.; Pereira-Lancha L. O; Lancha Jr A. Protein turnover, amino acid requirements and recommendations for athletes and active populations. Braz J Med Biol Res Num. 10 2012

27-Bröer S. Amino acid transport across mammalian intestinal and renal epithelia. Physiological. Review. V. 88, Núm. 1, pg. 249-286 2008

28-Balage M.; Dardevet D. Long-term effects of leucine supplementation on body composition. Current Opinion Clinical Nutrition Metabolism Care, v. 13, Núm. 3, pg. 265-70 2010

29-Tang J. ;Moore D. R.; Kujbida G. W.; Tarnopolsky M. A.; Phillips S. M. Ingestion of whey hydrolysate, casein, or soy protein isolate: effects on mixed muscle protein synthesis at rest and following resistance exercise in young men. J Appl Physiol 107 pg 987-992 2009

30-Tipton K.D., Elliott T.A.; Cree M.G.; Wolf S.E.; Sanford A.P.; Wolfe R.R. Ingestion of casein and whey proteins result in muscle anabolism after resistance exercise. Med Sci Sports Exerc Num.36 pg 2073-2081 2004

31-Aragon A.A.; Schoenfeld B. J. Nutrient timing revisited: is there a post-exercise anabolic window? Journal of the International Society of Sports Nutrition 10:5 2013

32-Walker D. K; Dickinson J. M.; Timmerman K. L. ; Drummond M. J.; Reidy P. T.; Fry C. S.; Gundermann D. M; et al. Exercise, Amino Acids 
and Aging in the Control of Human Muscle Protein Synthesis Med Sci Sports Exerc. Num. 43(12) pg. 2249-2258 2011

33-Tipton K. D.; Ferrando A. A.; Phillips S. M.; Doyle D. J.; Wolfe R. R.Postexercise net protein synthesis in human muscle from orally administered amino acids. American Journal of Physiology Num. 276 pg. E628-634 1999

34-Cribb P. J.; Hayes A. Effects of supplement timing and resistance exercise on skeletal muscle hypertrophy. Medicine and Science in Sports Exercise, Num. 11 pg. 1918-1925 2006

35-Koopman R. Role of amino acids and peptides in the molecular signaling in skeletal muscle after resistance exercise. International Journal of Sport Nutrition and Exercise Metabolism. Num. 17 pg. S47S57 2007

36-Phillips S. M. Protein Requirements and Supplementation in Strength Sports. Nutrition Num. 20 pg. 689-695 2004

37-Esmarck B.; Andersen J. L.; Olsen S.; Richter E. A.; Mizuno M.; Kjaer M. Timing of postexercise protein intake is important for muscle hypertrophy with resistance training in elderly humans. Journal of Physiology. Num. 535 pg. 301-311 2001

38-Rowlands D.; Thorp R.; Rossler K.; Graham D.; Rockell M. Efect of protein-rich feeding on recovery after intense exercise. Int J Sport Nutr Exerc Metab Num. 17 pg. 521-543 2007

39-Jentjens R.; Jeukendrup A. Determinants of post exericse glycogen synthesis during short term recovery. Sports Med Num. 33(2) pg. 117-144 2003

40-Rauch H.; Gibson A.; Lambert E.; Noakes T. A signalling role for muscle glycogen in the regulation of pace during prolonged exercise. Brit J Sport Med Num. 39 pg. 34-38 2005

41-Westerblad H.; Bruton J.D.; Katz A. Skeletal muscle: energy metabolism, fiber types, fatigue and adaptability. Exp Cell Res Num. 316(18) pg. 3093-3099 2010

42-Hill K. M.; Stathis C. G.; Grinfeld E.; Hayes A.; McAinch A. J. Coingestion of carbohydrate and whey protein isolates enhance PGC-1 $\alpha$ mRNA expression: a randomised, single blind, cross over study. Journal of the International Society of Sports Nutrition 10:8 2013

43-Akimoto T.; Pohnert S.; Li P.; Zhang M.; Gumbs C.; Rosenberg P.;Williams R.; et al. Exercise stimulates Pgc-1alpha transcription in skeletal muscle through activation of the p38 MAPK pathway. J Biol Chem Num. 280 pg. 19587-19593 2005

44-Glass D.J. Skeletal muscle hypertrophy and atrophy signaling pathways. Int J Biochem Cell Biol Num. 37 pg. 1974-1984 2005

45-Yan Z.; Okutsu M.; Akhtar Y. N; Okutsu, Yasir N.; Lira A. V. Regulation of exercise-induced fiber type transformation, muscle mitochondrial biogenesis, and angiogenesis in skeletal. J Appl Physiol Num. 110 pg. 264-274 2011

46-Coffey, V.G.; Hawley, J.A.The Specificity of Training: New Insights from Molecular Biology. Invited Review for Inaugural Issue. Malaysian Journal of Sport Science and Recreation. Num. (1) pg. 1-15 2005

47-Cermak N. M.; Res P. T.; de Groot L.; Saris W. H.; van Loon L. Protein supplementation augments the adaptive response of skeletal muscle to resistance-type exercise training: a meta-analysis. Am J Clin Nutr Num. 96 pg. 1454-64 2012

48-ECSS - 18th Annual Congress of the European College of Sport Science 2013

49-H. Nicastro; da Luz C. R.; Chaves D. S.; L. R. Bechara; Voltarelli V. ; Rogero M. M.; et al. Does Branched-Chain Amino Acids Supplementation Modulate SkeletalMuscle Remodeling through Inflammation Modulation? PossibleMechanisms of Action. Journal of Nutrition and Metabolism 2012

50-Dreyer H. C.; Drummond M. J.; Pennings B.; Fujita S.; Erin L.; Glynn; et al. Leucine-enriched essential amino acid and carbohydrate ingestion following resistance exercise enhances mTOR signaling and protein synthesis in human muscle. Am J Physiol Endocrinol Metab. Num. 294(2) pg. 3924002008

51-Churchward-Venne T. A.; Burd N. A.; Phillips S. M. Nutritional regulation of muscle protein synthesis with resistance exercise: strategies to enhance anabolism. Nutrition \& Metabolism 9:40 2012 52-Koopman R. ; Anton J. M.; Wagenmakers R.; Manders J.; Zorenc A.; Senden J.; et al. Combined ingestion of protein and free leucine with carbohydrate increases postexercise muscle protein synthesis in vivo in male subjects. American Journal of Physiology - Endocrinology and Metabolism. Num. 288 pg. E645-E653 2005

53-Campos-Ferraz P.L.; Bozza T.; Nicastro H.; Lancha A.H. Jr. Distinct effects of leucine or a mixture of the branched-chain amino acids (leucine, isoleucine, and valine) supplementation on resistance to fatigue, and muscle and liver-glycogen degradation, in trained rats. Nutrition. Num. 29(11-12) pg. 1388-94 2013

54-Katsanos C. S.; Kobayashi H.; Sheffield-Moore M.; Aarsland A.; Wolfe R. A high proportion of leucine is required for optimal stimulation of the rate of muscle protein synthesis by essential amino acids in the elderly. Am J Physiol Endocrinol Metab Num. 291 pg. E381-E387 2006

55-Garlick P.J. The role of leucine in the regulation of protein metabolism. J Nutrition Num. 135 pg. 1553S-1556S 2005

56-Terjung R.L.; Clarkson P.; Eichner E.R.; Greenhaff P.L.; Hespel P.J.; Israel R.G. The physiological and health effects of oral creatine supplementation. Med Sci Sports Exerc Num. 32 pg. 706-17 2000

57-Harris R.; Soderlund K.; Hultman E. Elevation of creatine in resting and exercised muscle of normal subjects by creatine supplementation. Clin Science Num. 83 pg. 367-74 1992

58-Stone M.H.; Sanborn K.; Smith L.L.; O’Bryant H.S.; Hoke T.; Utter A.C.,.Effects of in-season ( 5 weeks) creatine and pyruvate supplementation on anaerobic performance and body composition in American football players. Int J Sport Nutr Num. 9 pg. 146-65 1999 59-Buford T. W.; Kreider R.B.; Stout J. R.; Greenwood M.; Campbell B.; Spano M.; et al. International Society of Sports Nutrition position stand: creatine supplementation and exercise. Journal of the International Society of Sports Nutrition 4:6 2007

60-Gualano B.; Acquesta F. M.; Ugrinowitsch C.; Tricoli V.; Serrão J. C.; A. H. Lancha Junior. Effects of Creatine Supplementation on Strength and Muscle Hypertrophy: Current Concepts. Rev Bras Med Esporte. Vol. 16, Num 32010

61-Kreider R.B.; Willoughby D.; Greenwood M.; Parise G.; Payne E.; Tarnopolsky M.Effects of serum creatine supplementation on muscle creatine and phosphagen levels. J Exerc Physio Online Num. 6 pg. 24332006

62-Nissen S.L.; Sharp R.L. Effect of dietary supplements on lean mass and strength gains with resistance exercise: a meta-analisys. J Appl Physiol Num. 94 pg. 651-7 2003

63-Branch J.D. Effect of creatine supplementation on body composition and performance: a meta-analysis. Int J Sport Nutr Exerc Metab Num. 13 pg. 198-26 2003

64-Carvalho T.; Rodrigues T.; Meyer F.; Lancha Junior A.H.; De rose E.H. Modificações dietéticas, reposição hídrica, su-plementos alimentares e drogas: comprovação de ação ergogênica e potenciais riscos para a saúde. Rev Bras Med Esporte Num. 9 pg. 43-56 2003 65-Souza Júnior T.P.; Dubas J. P.; Pereira B.; de Oliveira P. R. Suplementação de creatina e treinamento de força: alterações na resultante de força máxima dinâmica e variáveis antropométricas em universitários submetidos a oito semanas de treinamento de força (hipertrofia). Rev Bras Med Esporte Vol. 13, Num. 52007

66-Hespel P.; Op't Eijnde B.; Van Leemputte M.; Urso B.; Greenhaff P.L.; Labarque V.; Dymarkowski S.; et al. Oral creatine supplementation facilitates the rehabilitation of disuse atrophy and alters the expression of muscle myogenic factors in humans. Journal of Physiology. Num. 15 pg. 625-33 2001

67-Kley R.A.; Vorgerd M.; Tarnopolsky M.A. Creatine for treating muscle disorders. Cochrane Database Syst Rev. 24;(1):CD004760 2007

68-Poortmans J.R.; Francaux M. Adverse effects of creatine supplementation: fact or fiction? Sports Med. Num. 30 pg. 155-70 2000

69-Kreider R.B.; Melton C.; Rasmussen C.J.; Greenwood M.; Lancaster S.; Cantler E.C. Long-term creatine supplementation does not significantly affect clinical markers of health in athletes. Mol Cell Biochem. Num. 244 pg. 95-104 2003

70-Gualano B.; Ugrinowitsch C.; Seguro A. C.; Lancha Junior A. H. Does Creatine Supplementation Harm Renal Function? Rev Bras Med Esporte. Vol. 14, Num. 2008

71-Gualano B.; Ugrinowitsch C.; Novaes R.B.; Artioli G.G.; Shimizu M.H.; Seguro A.C.; et al.; Effects of creatine supplementation on 


\section{REVISTA MÉDICA DA UFPR}

renal function: a randomized, double-blind, placebo-controlled clinical trial. Eur J Appl Physiol. Num. 103(1) pg. 33-40 2008

72-Gualano B.; Ferreira D.C.; Sapienza M.T.; Seguro A.C.; Lancha A.H. Jr. Effect of short-term, high-dose creatine supplementation on measured GFR in a young man with a single kidney. Am J Kidney Dis Nu. 55 pg. e7-e9 2009

73-Gualano B.; de Salles P.V.; Roschel H.; Artioli G.G.; Neves M. Jr; de Sá Pinto A.L., et al. Creatine in type 2 diabetes: A randomized, doubleblind, placebo-controlled trial. Med Sci Sports Exerc Num 43 pg. 770-778 2011

74-Jones T. E; Stephenson K. W.; King J. G.; Knight K. R.; Marshall T. L.; Scott W. B. Sarcopenia - Mechanisms and Treatments. Journal of Geriatric Physical Therapy Num. 322009

75-Sayer A. A; Robinson S; Patel H.; Shavlakadze T.; Cooper C.; Ground M. D. New horizons in the pathogenesis, diagnosis and management of sarcopenia. Age and Ageing Num. 42 pg. 145-150 2013

76-Burton L. A.; Sumukadas D.; Optimal management of sarcopenia. Clinical Interventions in Aging Num. 5 pg. 217-228 2010

77-Cruz-Jentoft A.; Baeyens J. P.; Bauer J. M.; Boirie Y.; Ederholm C.; Landi F.; Martin F.; et al. European Consensus on Definition and Diagnosis. Age and Ageing. Num. 39 pg. 412-423 2010

78-Sumukadas D.; Witham M. D.; Struthers A.; McMurdo M. T. Effect of perindopril on physical function in elderly people with functional impairment: a randomized controlled trial. CMAJ. 177(8) 2007

79-Gillespie L.D.; Robertson M.C.; Gillespie W.J. Interventions for preventing falls in older people living in the community. Cochrane Database Syst Rev. 3:CD007146 2009

80-Hughes V.A.; Frontera W.R.; Wood M.; Evans W.J.; Dallal G.E.; Roubenoff R.; et al. Longitudinal muscle strength changes in older adults: influence of muscle mass, physical activity, and health. J Gerontol A Biol Sci Med Sci 56: B209-B217 2001

81-Kumar V.; Selby A.; Rankin D.; Patel R.; Atherton P.; Hildebrandt W.; et al. Age-related differences in the dose-response relationship of muscle protein synthesis to resistance exercise in young and old men. J Physiol Num. 587 pg. 211-217 2009

82-Volpi E.; Kobayashi H.; Sheffield-Moore M.; Mittendorfer B.; Wolfe R.R. Essential amino acids are primarily responsible for the amino acid stimulation of muscle protein anabolism in healthy elderly adults. Am J Clin

Nutr Num. 78 pg. 250-258 2003.

83-Dreyer H. C.; Drummond M. J.; Pennings B.; Fujita S.; Glynn E. L.; Chinkes D. L.; et al. Leucine-enriched essential amino acid and carbohydrate ingestion following resistance exercise enhances mTOR signaling and protein synthesis in human muscle. Am J Physiol Endocrinol Metab. Num. 294(2) pg. 392-400 2008

84-Drummond M.J.; Fujita S.; Takash A.; Dreyer H.C.; Volpi E., Rasmussen B.B. Human muscle gene expression following resistance exercise and blood flow restriction. Med Sci Sports Exerc Num. 40 pg. 691-698 2008

85-Fujita S.; Abe T.; Drummond M.J.; Cadenas J.G.; Dreyer H.C.; Sato $\mathrm{Y}$; ; et al. Blood flow restriction during low-intensity resistance exercise increases S6K1 phosphorylation and muscle protein synthesis. J Appl Physiol Num. 103 pg. 903-910 2007.

86-Liu L.; Cash T.P.; Jones R.G.; Keith B.; Thompson C.B.; Simon M.C. Hypoxia-induced energy stress regulates mRNA translation and cell growth. Mol Cell Num. 21 pg. 521-531 2006

87-Fry C. S.; Glynn E. L.; Drummond M. J.; Timmerman K. L.; Fujita S; Abe T.; et al. Blood flow restriction exercise stimulates mTORC1 signaling and muscle protein synthesis in older men. J Appl Physiol Num. 108 pg. 1199-1209 2010

88-Breen L.; Phillips S. M. Skeletal muscle protein metabolism in the elderly: Interventions to counteract the 'anabolic resistance' of ageing. Nutrition \& Metabolism 8:68 2011

89-Paddon-Jones D.; Rasmussen B. B. Dietary protein recommendations and the prevention of Sarcopenia. Curr Opin Clin Nutr Metab Care Num 12(1) pg. 86-90 2009

90-Volpi E; Mittendorfer B.; Rasmussen B.B.; Wolfe R.R. The response of muscle protein anabolism to combined hyperaminoacidemia and glucose induced hyperinsulinemia is impaired in the elderly. J Clin Endocrinol Metab Num. 85 pg. 4481-4490 2000
91-Donges C. E.; Burd N. A.; Duffield R.; Smith G. C.; West D; Short M. J.; et al. Concurrent resistance and aerobic exercise stimulates both myofibrillar and mitochondrial protein synthesis in sedentary middleaged men. J Appl Physiol Num. 112 pg. 1992-2001 2012

92-Phillips B.E.; Williams J.P.; Gustafsson T.; Bouchard C.; Rankinen B.; Atherton A. Molecular Networks of Human Muscle Adaptation to Exercise and Age Genomic Features of Muscle Age Vol 9 Issue 32013 93-Katsanos C.S.; Kobayashi H.; Sheffield-Moore M.; Wolf R.R. A high proportion of leucine is required for optimal stimulation of the rate of muscle protein synthesis by essential amino acids in the elderly. Am J Physiol Endocrinol Metab Num. 291 pg. 381-E387 2006

94-Rieu I.; Sornet C.; Bayle G.; Prugnaud J.; Pouyet C.; Balage M.; et al. Leucine-supplemented meal feeding for ten days beneficially affects postprandial muscle protein synthesis in old rats. J Nutr Num. 133 pg. 1198-1205 2003

95-Dalbo V.J.; Roberts M.D.; Lockwood C. M.; Tucker P.S.; Kreider R.B.; Cherksick K. The effects of age on skeletal muscle and the phosphocreatine energy system: can creatine supplementation help older adults. Dynamic Medicine 8:6 2009

96-Gualano B.; Artioli G.G.; Poortmans J.R.; Lancha Junior A.H. Exploring the therapeutic role of creatine supplementation. Amino Acids Num. 38(1) pg. 31-44 2009

97-Gualano B.; Novaes R.B.; Artioli G.G.; Freire T.O.; Coelho D.F.; Scagliusi F.B.; et al. Effects of creatine supplementation on glucose tolerance and insulin sensitivity in sedentary healthy males undergoing aerobic training. Amino Acids Num. 34(2) pg. 245-250 2008 